\title{
On Notations for Conic Hulls and Related Considerations on Tangent Cones
}

\author{
Giorgio Giorgi \\ Correspondence: Giorgio Giorgi, Department of Economics and Management, Via S. Felice, 5-27100, Pavia, Italy
}

Received: February 24, 2021 Accepted: April 1, 2021 Online Published: April 14, 2021

doi:10.5539/jmr.v13n3p13

URL: https://doi.org/10.5539/jmr.v13n3p13

\begin{abstract}
We propose two different notations for cones generated by a set and for convex cones generated by a set, usually denoted by a same notation. We make some remarks on the Bouligand tangent cone and on the Clarke tangent cone for star-shaped sets and for locally convex sets. We give some applications of these remarks to a differentiable optimization problem with an abstract constraint.
\end{abstract}

Keywords: conic hulls, convex conic hulls, generated cones, star-shaped sets, Bouligand tangent cone, Clarke tangent cone

Mathematics Subject Classification (2010): 90C30

\section{Introduction}

In the first part of this paper, i. e. in Section 2, we recall some basic definitions concerning the various types of combinations of vectors of a set $S \subset \mathbb{R}^{n}$; we point out that in the current literature it is often used the same notation to denote the conic hull of $S$ and the convex conic hull of $S$, i. e. cone $(S)$, which may generate some confusions and misunderstandings. We propose two different notations and make some remarks concerning some particular cases. In the second part of this paper, i. e. in Section 3, we make some remarks on the Bouligand tangent cone (or contingent cone) to $S$ at $x^{0} \in S$ and on the Clarke tangent cone to $S$ at $x^{0} \in S$, when the related set $S$ is convex or locally convex at $x^{0} \in S$ or star-shaped at $x^{0} \in S$. In Section 4 we give some applications of the previous remarks to a differentiable optimization problem with an abstract constraint.

\section{Conic Hulls and Convex Conic Hulls}

We recall that a set $K \subset \mathbb{R}^{n}$ is a cone with vertex at the origin if

$$
x \in K \Longrightarrow \lambda x \in K, \quad \forall \lambda>0 .
$$

Many authors, however, do include the vertex in the cone by letting $\lambda \geqq 0$ in the definition. It may be useful to accept both definitions, in order to have more flexibility in the various cases and problems encountered in treating this topic. It is well-known that a cone $K \subset \mathbb{R}^{n}$ is convex if and only if

$$
x, y \in K \Longrightarrow x+y \in K
$$

Let $x^{1}, x^{2}, \ldots, x^{k}$ be vectors of $\mathbb{R}^{n}$. We recall that a linear combination of the vectors $x^{1}, x^{2}, \ldots, x^{k}$ is any expression of the form

$$
\sum_{i=1}^{k} \alpha_{i} x^{i}
$$

where each $\alpha_{i} \in \mathbb{R}$.

When $\sum_{i=1}^{k} \alpha_{i}=1$, the linear combination (1) is called affine combination of the vectors $x^{1}, x^{2}, \ldots, x^{k}$.

When it holds that each $\alpha_{i} \geqq 0$, the linear combination (1) is called convex conic combination or nonnegative combination of the vectors $x^{1}, x^{2}, \ldots, x^{k}$.

When in (1) it holds that each $\alpha_{i} \geqq 0$ and $\sum_{i=1}^{k} \alpha_{i}=1$, then the linear combination (1) is called a convex combination of the vectors $x^{1}, x^{2}, \ldots, x^{k}$.

- The collection of all linear combinations of vectors of $S$ is said to be the linear space generated by $S$ or linear span 
of $S$ or also linear hull of $S$ and it is denoted by

$$
\begin{gathered}
\operatorname{lin}(S) \text { or } \operatorname{span}(S): \\
\operatorname{lin}(S)=\left\{\sum_{i=1}^{k} \alpha_{i} x^{i}: \text { each } x^{i} \in S, \text { each } \alpha_{i} \in \mathbb{R}, k \in \mathbb{N}\right\}
\end{gathered}
$$

It can be proved that $\operatorname{lin}(S)$ is the smallest linear space of $\mathbb{R}^{n}$ containing $S$.

- The collection of all affine combinations of the vectors of $S$ is called affine hull of $S$ and it is denoted by

$$
\begin{gathered}
\operatorname{aff}(S): \\
\operatorname{aff}(S)=\left\{\sum_{i=1}^{k} \alpha_{i} x^{i}: \text { each } x^{i} \in S, \text { each } \alpha_{i} \in \mathbb{R}, \sum_{i=1}^{k} \alpha_{i}=1, k \in \mathbb{N}\right\} .
\end{gathered}
$$

It can be proved that $\operatorname{aff}(S)$ is the smallest affine subspace of $\mathbb{R}^{n}$ containing $S$.

- The collection of all convex conic combinations of the vectors of $S$ is called convex conic hull of $S$ or convex cone generated by $S$ or convex cone spanned by $S$ and it is denoted, in the present paper, by

$$
\begin{gathered}
\operatorname{cone}(S): \\
\operatorname{cone}(S)=\left\{\sum_{i=1}^{k} \alpha_{i} x^{i}, \text { each } x^{i} \in S, \text { each } \alpha_{i} \geqq 0, k \in \mathbb{N}\right\} .
\end{gathered}
$$

Some authors (e. g. Bazaraa and Shetty (1976)) require that the coefficients $\alpha_{i}, i=1, \ldots, k$, are not all zero, i. e. $\sum_{i=1}^{k} \alpha_{i}>0$. It can be proved that $\operatorname{cone}(S)$ is the smallest convex cone of $\mathbb{R}^{n}$ containing $S$ (i. e. the intersection of all convex cones in $\mathbb{R}^{n}$ containing $S$ ).

- The collection of all convex combinations of the vectors of $S$ is called the convex hull of $S$ and it is denoted by

$$
\operatorname{conv}(S) \text { or } \operatorname{co}(S) \text { : }
$$

$$
\operatorname{conv}(S)=\left\{\sum_{i=1}^{k} \alpha_{i} x^{i}, \text { each } x^{i} \in S, \text { each } \alpha_{i} \geqq 0, \sum_{i=1}^{k} \alpha_{i}=1, k \in \mathbb{N}\right\} .
$$

It can be proved that $\operatorname{conv}(S)$ is the smallest convex set of $\mathbb{R}^{n}$ containing $S$ (i. e. the intersection of all convex sets in $\mathbb{R}^{n}$ containing $S$ ).

We note that if $S$ is any nonempty subset of $\mathbb{R}^{n}$, we have

$$
\begin{aligned}
& S \quad \subset \operatorname{conv}(S) \subset \operatorname{aff}(S) \subset \operatorname{lin}(S) ; \\
& S \quad \subset \operatorname{conv}(S) \subset \operatorname{cone}(S) \subset \operatorname{lin}(S) .
\end{aligned}
$$

Moreover:

a) $\quad S$ is a linear subspace of $\mathbb{R}^{n}$ if and only if $S=\operatorname{lin}(S)$;

b) $\quad S$ is an affine set of $\mathbb{R}^{n}$ if and only if $S=\operatorname{aff}(S)$;

c) $\quad S$ is a convex cone of $\mathbb{R}^{n}$ with vertex at $0 \in S$ if and only if $S=\operatorname{cone}(S)$;

d) $\quad S$ is a convex set of $\mathbb{R}^{n}$ if and only if $S=\operatorname{conv}(S)$.

The notation "cone $(S)$ " is widely used, however some authors use other notations: for example Jeter (1986) uses the notation coni $(S)$, whereas Dhara and Dutta (2012) use the notation cone co $(S)$, to denote the convex cone generated by $S$. Some authors call the convex cone generated (or spanned) by $S$, the positive hull of $S$.

We remark that cone $(S)$ need not be a closed set, even if $S$ is compact. However, it can be proved that cone $(S)$ is closed (and convex) in special cases, such as when $S$ is finite. We speak in this case of finite cone or finitely generated cone, i. e. the closed and convex cone generated by a finite set $C=\left\{a^{1}, a^{2}, \ldots, a^{m}\right\}$ of vectors in $\mathbb{R}^{n}$ :

$$
\operatorname{cone}(C)=\left\{x \in \mathbb{R}^{n}: x=\sum_{i=1}^{m} \alpha_{i} a^{i}, \alpha_{i} \geqq 0, i=1, \ldots, m\right\} .
$$


The vectors $a^{1}, a^{2}, \ldots, a^{m}$ of $\mathbb{R}^{n}$ are said to be the generators of cone $(C)$. If $a^{i} \equiv A_{i}, i$-th row of a matrix $A$ of order $(m, n)$, then

$$
\text { cone }\left(a^{1}, a^{2}, \ldots, a^{m}\right)=\left\{x \in \mathbb{R}^{n}: \mu A=x, \mu \geqq 0\right\} .
$$

The proof that a finitely generated cone is closed is basic in proving the well-known Farkas' lemma, by means of a separation theorem between convex sets, even if several authors give this property for granted and skip the related proof. Some authors use, for the case of a finite number of generators, instead of the notation

$$
\text { cone }\left(a^{1}, a^{2}, \ldots, a^{m}\right),
$$

the notation

$$
\operatorname{pos}\left(a^{1}, a^{2}, \ldots, a^{m}\right)
$$

and, if $a^{1}, a^{2}, \ldots, a^{m}$ are the $m$ rows of a matrix $A$ of order $(m, n)$, also the notation

$$
\left(\mathbb{R}_{+}^{m}\right) A .
$$

It is well-known that a cone described by

$$
K=\left\{x \in \mathbb{R}^{n}: B x \leqq 0\right\},
$$

where $B$ is a matrix of order $(m, n)$, is called a polyhedral (convex) cone. The Minkowski-Weyl Theorem states that a cone is polyhedral if and only if it is finitely generated. See, e. g., Bertsekas (1999), Florenzano and Le Van (2001), Hiriart-Urruty and Lemarechal (1993), Rockafellar (1970).

In the literature it is often introduced the conic hull of $S \subset \mathbb{R}^{n}$ or radial hull of $S$ as the smallest cone of $\mathbb{R}^{n}$ (not necessarily convex!) which contains $S$. Unfortunately this cone is almost always denoted by $\operatorname{cone}(S)$, which can generate some confusions and misunderstandings. As this cone is given by the union of all rays starting from the origin and passing through the element $x \in S$, we propose the notation $\operatorname{ray}(S)$ :

$$
\operatorname{ray}(S)=\{y: y=\lambda x, x \in S, \lambda \geqq 0\} .
$$

Some authors require $\lambda>0$ and some authors use the notation $\mathbb{R}_{+}(S)$.

Furthermore, some authors speak, for the present case, of cone generated (or spanned) by $S$, however this can be misleading, as in the definition of cone $(S)$ the term "convex" is often omitted. Note that the nonempty set $S \subset \mathbb{R}^{n}$ is a cone if and only if $\operatorname{ray}(S)=S$.

The basic relation between cone $(S)$ and $\operatorname{ray}(S)$ is given by the following result.

Theorem 1. If $S \subset \mathbb{R}^{n}$ is a nonempty convex set, then

$$
\operatorname{cone}(S)=\operatorname{ray}(S) .
$$

See, e. g., Bazaraa and Shetty (1976).

Moreover, it holds, with $S \subset \mathbb{R}^{n}$ :

$$
\operatorname{cone}(S)=\operatorname{ray}(\operatorname{conv}(S))=\operatorname{conv}(\operatorname{ray}(S)) .
$$

It is sometimes also useful to consider cones and convex cones generated by a set $S \subset \mathbb{R}^{n}$, with reference to a point $x^{0} \in S$ (or also with reference to a point $x^{0} \in \operatorname{cl}(S)$ or also $x^{0} \in \mathbb{R}^{n}$ ). Let $x^{0} \in S$, with $S$ nonempty set of $\mathbb{R}^{n}$. Then $\operatorname{ray}\left(S, x^{0}\right)$ is the smallest cone of $\mathbb{R}^{n}$ which contains $S-x^{0}$ and cone $\left(S, x^{0}\right)$ is the smallest convex cone of $\mathbb{R}^{n}$ which contains $S-x^{0}$, i. e., with $x^{0} \in S$,

$$
\begin{gathered}
\operatorname{ray}\left(S, x^{0}\right)=\operatorname{ray}\left(S-x^{0}\right)=\left\{y: \lambda\left(x-x^{0}\right), x \in S, \lambda \geqq 0\right\} ; \\
\operatorname{cone}\left(S, x^{0}\right)=\operatorname{cone}\left(S-x^{0}\right) .
\end{gathered}
$$

These cones are also called, respectively, the cone generated by $S$ at $x^{0}$ (or from $x^{0}$ ) and the convex cone generated by $S$ at $x^{0}\left(\right.$ or from $\left.x^{0}\right)$. The cone generated by $S$ at $x^{0}$ is called by Palata (1989) the projection cone of $S$ at $x^{0}$. This cone may be viewed as a (very) rough approximation of the set $S$ in a neighborhood of $x^{0} \in S$. In the next section we shall consider two more "refined" local cone approximations of the set $S$ at $x^{0} \in S$. 


\section{Tangent Cones to Convex Sets, Locally Convex Sets and Star-Shaped Sets}

There are several local cone approximations of a set $S \subset \mathbb{R}^{n}$ at $x^{0} \in S$, which better than $\operatorname{ray}\left(S, x^{0}\right)$ "represent" the structure of $S$ around the point $x^{0} \in S$. For a survey, see, e. g., Aubin and Frankowska (1990), Bazaraa and Shetty (1976), Giorgi and Guerraggio (1992, 2002), Palata (1989).

Definition 1. A sequence $\left\{x^{k}\right\} \subset \mathbb{R}^{n} \backslash\left\{x^{0}\right\}$, with $x^{k} \longrightarrow x^{0}$ is called tangentially convergent in the direction $y \in \mathbb{R}^{n}$ to the point $x^{0}$ if

$$
\lim _{k \rightarrow+\infty} \frac{x^{k}-x^{0}}{\left\|x^{k}-x^{0}\right\|}=y
$$

and we write $x^{k} \stackrel{y}{\longrightarrow} x^{0}$.

Obviously any convergent sequence $x^{k} \longrightarrow x^{0}$ (with $x^{k} \neq x^{0}$ for all $k$ ) contains at least a tangentially convergent subsequence. The set of all directions $y$ for which there exists a feasible sequence $\left\{x^{k}\right\} \subset S$, with $S \subset \mathbb{R}^{n}$, tangentially convergent to $x^{0} \in S$, form a cone which is a local cone approximation at $x^{0}$ of the set $S$.

Definition 2. Let $S \subset \mathbb{R}^{n}$ and $x^{0} \in S$; the cone

$$
T\left(S, x^{0}\right)=\left\{\lambda y \in \mathbb{R}^{n}: \exists\left\{x^{k}\right\} \subset S, x^{k} \stackrel{y}{\longrightarrow} x^{0}, \lambda \geqq 0\right\}
$$

is called Bouligand tangent cone or contingent cone to the set $S$ at the point $x^{0}$. If $x^{0}$ is an isolated point of $S$, we set $T\left(S, x^{0}\right)=\{0\}$.

Other equivalent characterizations of $T\left(S, x^{0}\right)$ are the following ones:

$$
\begin{gathered}
T\left(S, x^{0}\right)=\left\{\begin{array}{c}
y \in \mathbb{R}^{n}: \exists\left\{x^{k}\right\} \subset S, \lim _{k \rightarrow+\infty} x^{k}=x^{0}, \exists\left\{\lambda_{k}\right\} \subset \mathbb{R}_{+} \\
\text {such that } y=\lim _{k \rightarrow+\infty} \lambda_{k}\left(x^{k}-x^{0}\right)
\end{array}\right\} ; \\
T\left(S, x^{0}\right)=\left\{y \in \mathbb{R}^{n}: \exists\left\{y^{k}\right\} \longrightarrow y, \exists\left\{t_{k}\right\} \longrightarrow 0, \text { such that } x^{0}+t_{k} y^{k} \in S\right\} ; \\
T\left(S, x^{0}\right)=\left\{y \in \mathbb{R}^{n}: \forall N(y), \forall \lambda>0, \exists t \in(0, \lambda), \exists \bar{y} \in N(y) \text { such that } x^{0}+t \bar{y} \in S\right\} .
\end{gathered}
$$

Note that $T\left(S, x^{0}\right)$ is indeed a cone, closed, but not necessarily convex, with $0 \in T\left(S, x^{0}\right)$. We note also that:

i) $\quad T\left(S, x^{0}\right)$ depends only from the structure of $S$ in a neighborhood of $x^{0}$, that is

$$
T\left(S, x^{0}\right)=T\left(S \cap U\left(x^{0}\right), x^{0}\right)
$$

where $U\left(x^{0}\right)$ is any neighborhood of $x^{0}$ (the notion of "Bouligand tangent cone" is therefore an "infinitesimal notion").

ii) If $x^{0} \in \operatorname{int}(S)$, then $T\left(S, x^{0}\right)=\mathbb{R}^{n}$.

iii) $\quad T\left(S, x^{0}\right)=T\left(\bar{S}, x^{0}\right)$, where $\bar{S}=\operatorname{cl}(S)$.

iv) $\quad T\left(S_{1}, x^{0}\right) \subset T\left(S_{2}, x^{0}\right)$, if $x^{0} \in S_{1} \subset S_{2}$.

Moreover, we note that

$$
T\left(S, x^{0}\right) \subset \operatorname{cl}\left(\operatorname{ray}\left(S-x^{0}\right)\right),
$$

however if $S$ is a nonempty convex set, we have the following basic result.

Theorem 2. Let $S \subset \mathbb{R}^{n}$ be a nonempty convex set and let be $x^{0} \in S$, then

$$
T\left(S, x^{0}\right)=\operatorname{cl}\left(\operatorname{cone}\left(S-x^{0}\right)\right)=\operatorname{cl}\left(\operatorname{ray}\left(S-x^{0}\right)\right) .
$$

Therefore, if $S$ is a convex set, $T\left(S, x^{0}\right)$ is a closed convex cone.

Definition 3. Let $S \subset \mathbb{R}^{n}$ be a nonempty set and let $x^{0} \in S$. Then $S$ is said to be star-shaped at $x^{0}$ (or star-convex at $x^{0}$ or radially convex at $x^{0}$ ) if

$$
\lambda x^{0}+(1-\lambda) x \in S, \quad \forall x \in S, \forall \lambda \in[0,1] .
$$


Some authors require $x^{0} \in \operatorname{cl}(S)$ and $\lambda x^{0}+(1-\lambda) x \in S, \forall x \in S, \forall \lambda \in(0,1)$. It is evident that a convex set is star-shaped at every point $x^{0} \in S$ and conversely. We note, moreover, that the closure of a star-shaped set at $x^{0} \in S$ is a star-shaped set at $x^{0}$, but the interior of a star-shaped set at $x^{0}$ is not necessarily a star-shaped set. It can be proved the following result (see, e. g., Bazaraa and Shetty (1976)).

Theorem 3. Let Let $S \subset \mathbb{R}^{n}$ be a nonempty set and let be $x^{0} \in S$. Suppose that $S$ is star-shaped at $x^{0}$. Then

$$
T\left(S, x^{0}\right)=\operatorname{cl}\left(\operatorname{ray}\left(S, x^{0}\right)\right) .
$$

If we introduce the notion of (negative) polar cone $S^{*}$ of the nonempty set $S \subset \mathbb{R}^{n}$ :

$$
S^{*}=\left\{y \in \mathbb{R}^{n}: y x \leqq 0, \forall x \in S\right\},
$$

then it is not difficult to prove that if $S$ is convex at $x^{0} \in S$ (in particular if $S$ is a convex set), then $y \in\left(T\left(S, x^{0}\right)\right)^{*}$ if and only if $y\left(x-x^{0}\right) \leqq 0, \forall x \in S$. This fact will be used further to develop some considerations on optimality criteria for set-constrained minimization problems.

We have already remarked that in general $T\left(S, x^{0}\right)$ is not a convex cone. Guignard (1969) has introduced the following notion, in order to overcome this "defect", without assuming convexity of $S$. The closed convex hull of $T\left(S, x^{0}\right)$ is called the pseudotangent cone to $S$ at $x^{0}$ and is denoted $P\left(S, x^{0}\right)$ :

$$
P\left(S, x^{0}\right)=\operatorname{cl}\left(\operatorname{conv}\left(T\left(S, x^{0}\right)\right)\right) .
$$

In studying the sufficiency of first-order optimality conditions Guignard (1969) has introduced the concept of pseudoconvexity of a set $S \subset \mathbb{R}^{n}$ at a point $x^{0} \in S$.

Definition 4. A nonempty set $S \subset \mathbb{R}^{n}$ is said to be pseudoconvex at $x^{0} \in S$ if

$$
S-x^{0} \subset P\left(S, x^{0}\right) .
$$

It is easy to see that when $S$ is star-shaped at $x^{0}$, then $S$ is pseudoconvex at $x^{0}$ and that if $S$ is convex, then $S$ is pseudoconvex at $x^{0}$ for all $x^{0} \in S$. Guignard (1969) remarks that the union of several convex sets, the intersection of which is not empty, is pseudonvex at any point of this intersection. This is another example of a pseudoconvex set which is not necessarily convex.

Another definition that sometimes is encountered in the literature is the following one.

Definition 5. Let be given the nonempty set $S \subset \mathbb{R}^{n}$ and let be $x^{0} \in S$. Then $S$ is said to be locally convex at $x^{0}$ if there exists a neighborhood $V\left(x^{0}\right)$ such that the set $S \cap V\left(x^{0}\right)$ is convex.

We recall that the first-order local cone approximations depend only on the structure of the set $S$ near $x^{0}$. Hence, if $S \cap V\left(x^{0}\right)$ is a convex set, i. e. if $S$ is locally convex at $x^{0}$, then $T\left(S, x^{0}\right)$ is convex too and it will hold

$$
T\left(S, x^{0}\right)=\operatorname{cl}\left(\operatorname{cone}\left(S \cap V\left(x^{0}\right)\right), x^{0}\right) .
$$

It must be remarked that the cone in the right-hand side of this last equality is in general smaller than $\operatorname{cl}\left(\operatorname{cone}\left(S-x^{0}\right)\right)$. Simple examples can be created.

We have seen that one of the drawbacks of the Bouligand tangent cone is that it is not convex in general. To overcome this fact, Clarke (see, e. g., Clarke $(1975,1983))$ has introduced a cone which is always closed and convex. This cone can be represented in various ways, e. g., by means of sequences (see, e. g., Giorgi and Guerraggio (1992, 2002), Hiriart-Urruty (1979)).

Definition 6. Let be given the nonempty set $S \subset \mathbb{R}^{n}$ and let be $x^{0} \in S$. The cone

$$
T_{C}\left(S, x^{0}\right)=\left\{\begin{array}{l}
y \in \mathbb{R}^{n}: \forall t_{k} \longrightarrow 0^{+}, \forall x^{k} \longrightarrow x^{0}, \text { with }\left\{x^{k}\right\} \subset S, \\
\exists y^{k} \longrightarrow y, \text { such that } x^{k}+t_{k} y^{k} \in S
\end{array}\right\}
$$

is called Clarke tangent cone to $S$ at $x^{0}$. 
Theorem 4. For any nonempty set $S \subset \mathbb{R}^{n}$ the Clarke tangent cone is closed and convex. Moreover,

$$
T_{C}\left(S, x^{0}\right) \subset T\left(S, x^{0}\right) .
$$

If $S$ is a convex set, we have

$$
T_{C}\left(S, x^{0}\right)=T\left(S, x^{0}\right)=\operatorname{cl}\left(\operatorname{cone}\left(S, x^{0}\right)\right)=\operatorname{cl}\left(\operatorname{ray}\left(S, x^{0}\right)\right)
$$

Remark 1. The two cones $T_{C}\left(S, x^{0}\right)$ and $T\left(S, x^{0}\right)$ are equal (and convex!) also in the case $S$ is a locally convex set at $x^{0} \in S$. We have in this case

$$
T_{C}\left(S, x^{0}\right)=T\left(S, x^{0}\right)=\operatorname{cl}\left(\operatorname{cone}\left(S \cap V\left(x^{0}\right), x^{0}\right)\right) .
$$

In general, when $T_{C}\left(S, x^{0}\right)=T\left(S, x^{0}\right)$, we say that $S$ is Clarke regular at $x^{0}$ : see, e. g., Rockafellar and Wets (2009).

Remark 2. If $S \subset \mathbb{R}^{n}$ is star-shaped at $x^{0} \in S$, it is no longer true that $T_{C}\left(S, x^{0}\right)=T\left(S, x^{0}\right)$, as asserted in Palata (1989) and in Giorgi and Guerraggio (1992). When $S$ is star-shaped at $x^{0}$, we can assert, for example (see Bazaraa and Shetty (1976)), that the Bouligand tangent cone is equal to the Kuhn-Tucker tangent cone (called also cone of attainable directions), but not, as in Giorgi and Guerraggio (1994), that the Bouligand tangent cone is convex. If $S$ is locally convex at $x^{0}$, then the convexity of $T\left(S, x^{0}\right)$ follows, as previously explained. We take the opportunity to point out an oversight appearing in Hiriart-Urruty (1979) and in a footnote of R. Cambini (2002). These authors assume $S \subset \mathbb{R}^{n}$ to be locally convex at $x^{0} \in S$, but write the equality

$$
T_{C}\left(S, x^{0}\right)=T\left(S, x^{0}\right)=\operatorname{cl}\left(\operatorname{cone}\left(S, x^{0}\right)\right)
$$

instead of

$$
T_{C}\left(S, x^{0}\right)=T\left(S, x^{0}\right)=\operatorname{cl}\left(\operatorname{cone}\left(S \cap V\left(x^{0}\right), x^{0}\right)\right) .
$$

Summing up:

- $\quad$ If $S \subset \mathbb{R}^{n}$ is star-shaped at $x^{0} \in S$, then $S$ is pseudoconvex at $x^{0}$.

- If $S \subset \mathbb{R}^{n}$ is convex, then $S$ is locally convex at any $x^{0} \in S$.

- If $S \subset \mathbb{R}^{n}$ is locally convex at $x^{0} \in S$, then $T\left(S, x^{0}\right)=T_{C}\left(S, x^{0}\right)$ (and therefore $T\left(S, x^{0}\right)$ is a convex set).

\section{Applications to Optimization Problems}

Let us consider the following minimization problem with an abstract constraint:

$$
(P): \quad \min f(x), \quad x \in S,
$$

where $S \subset \mathbb{R}^{n}$ is a nonempty set and $f(x)$ is differentiable at a point $x^{0} \in S$.

With regard to problem $(P)$ the following result, due to Gould and Tolle (1971), Guignard (1969), Varaiya(1967) is of basic importance.

Theorem 5. If $x^{0} \in S$ is a local solution of $(P)$, then

$$
-\nabla f\left(x^{0}\right) \in\left(T\left(S, x^{0}\right)\right)^{*}
$$

i. e.

$$
\nabla f\left(x^{0}\right) y \geqq 0, \quad \forall y \in T\left(S, x^{0}\right) .
$$

The result obtained by Guignard (1969):

$$
-\nabla f\left(x^{0}\right) \in\left(P\left(S, x^{0}\right)\right)^{*}
$$

is not sharper than the thesis of Theorem 5 , because for any cone $C \subset \mathbb{R}^{n}$, it holds $C^{*}=(\operatorname{cl}(\operatorname{conv}(C)))^{*}$.

If $S$ is a nonempty convex set, relation (2) of Theorem 5 can be written as

$$
\nabla f\left(x^{0}\right)\left(x-x^{0}\right) \geqq 0, \quad \forall x \in S .
$$


On the grounds of what previously remarked on star-shaped sets, if $S$ is star-shaped at $x^{0} \in S$ and $x^{0}$ is a local solution of $(P)$, then (3) holds.

If (3) holds and $f(x)$ is pseudoconvex at $x^{0} \in S$ with respect to $S$, i. e. (see Mangasarian (1969)

$$
\nabla f\left(x^{0}\right)\left(x-x^{0}\right) \geqq 0 \Longrightarrow f(x) \geqq f\left(x^{0}\right), \quad \forall x \in S,
$$

then (3) becomes also a sufficient condition for the global optimality of $x^{0}$ for $(P)$.

It is easy to prove that the class of differentiable convex functions at $x^{0} \in S$ with respect to $S$, is strictly contained in the class of pseudoconvex functions at $x^{0} \in S$ with respect to $S$. Unfortunately, there are no inclusion relationships between quasi-convexity, semistrict quasiconvexity and pseudoconvexity at a point $x^{0} \in S$ with respect to $S$ (see Cambini and Martein (2009)). However, if $f(x)$ is continuous on the open set $S \subset \mathbb{R}^{n}$, and $f(x)$ is differentiable and quasiconvex at $x^{0} \in S$ with respect to $S$, with $\nabla f\left(x^{0}\right) \neq 0$, then $f(x)$ is pseudoconvex at $x^{0}$ with respect to $S$.

We remark that Guignard (1969) has proved that relation (2) is sufficient for the global optimality of $x^{0} \in S$ for $(P)$ if $f(x)$ is pseudoconvex at $x^{0}$ with respect to $S$ and $S$ is pseudoconvex at $x^{0}$. We note also that in absence of generalized convexity assumptions on $f(x)$, a stronger form of (2) is a sufficient optimality condition for $x^{0} \in S$ to be a local strict minimizer for $(P)$. The following result is due to Hestenes $(1966,1975)$.

Theorem 6. If for $x^{0} \in S$ it holds

$$
\nabla f\left(x^{0}\right) y>0, \quad \forall y \in T\left(S, x^{0}\right) \backslash\{0\},
$$

then $x^{0}$ is a strict local minimizer for $(P)$. More precisely, there exist a positive number $\alpha$ and a neighborhood $N\left(x^{0}\right)$ of $x^{0}$ such that

$$
f(x)-f\left(x^{0}\right) \geqq \alpha\left\|x-x^{0}\right\|, \quad \forall x \in N\left(x^{0}\right) \cap S .
$$

It must be noted that condition (4) requires for its application that $T\left(S, x^{0}\right)$ is a pointed cone, i. e. a cone which does not contain any line through the origin. Relation (5) in other words says that $x^{0}$ is a strict local minimizer of order 1 or strong local minimizer or sharp local minimizer (see, e. g., Still and Streng (1996), Polyak (1987)).

Another relationship between $(P)$ and the Bouligand tangent cone to $S$ at $x^{0} \in S$ is given in the following result (see, e. g., Giorgi (1985), Martein (1985)).

Theorem 7. If $x^{0} \in S$ is a local solution of $(P)$, then $x^{0}$ is also solution of the problem

$$
\min _{x \in T\left(S, x^{0}\right)}\left\{f\left(x^{0}\right)+\nabla f\left(x^{0}\right)\left(x-x^{0}\right)\right\} .
$$

The optimality conditions expressed by the previous results involve vectors belonging to the Bouligand tangent cone $T\left(S, x^{0}\right)$. In general, however, there is no relationship between the optimality of $x^{0} \in S$ for $(P)$ and for the problem

$$
\left(P_{1}\right): \quad \min f(x), \quad x \in T\left(S, x^{0}\right) \cap S .
$$

For example, consider the problem

$$
\left\{\begin{array}{l}
\min _{x \in S} f(x, y)=y \\
S=\left\{(x, y) \in \mathbb{R}^{2}: x^{3}+y \geqq 0\right\} .
\end{array}\right.
$$

Here $(P)$ has no solution, whereas $x^{0}=(0,0)$ is a local minimizar for $\left(P_{1}\right)$.

If we consider the problem

$$
\left\{\begin{array}{l}
\min _{x \in S} f(x, y)=y-x^{2} \\
S=\left\{(x, y) \in \mathbb{R}^{2}: x^{2}-y \leqq 0\right\},
\end{array}\right.
$$

the point $x^{0}=(0,0)$ is a local minimizer for $(P)$, whereas the problem $\left(P_{1}\right)$ has no solution.

The author thanks an anonymous referee for some useful suggestions. 


\section{References}

Aubin, J. P., \& Frankowska, H. (1990). Set-Valued Analysis. Birkhäuser, Boston.

Bazaraa, M. S., \& Shetty, C. M. (1976). Foundations of Optimization. Springer Verlag, New York. https://doi.org/10.1007/978-3-642-48294-6

Bertsekas, D. P. (1999). Nonlinear Programming (2nd ed.). Athena Scientific, Belmont, Mass.

CAMBINI, R. (2002). Developments on U-regularity conditions; in G. P. Crespi and others (Eds.), Optimization in Economics, Finance and Industry, Datanova Editrice, Milan, 103-117.

Cambini, A., \& Martein, L. (2009). Generalized Convexity and Optimization. Theory and Applications. Springer, Berlin.

CLARKE, F. H. (1975). Generalized gradients and applications, Trans. Am. Math. Soc., 205, 247-262. https://doi.org/10.1090/S0002-9947-1975-0367131-6

Clarke, F. H. (1983). Optimization and Nonsmooth Analysis, J. Wiley, New York.

Dhara, A., \& Dutta, J. (2012). Optimality Conditions in Convex Optimization. A Finite-Dimensional View, CRC Press, Boca Raton, London, New York. https://doi.org/10.1201/b11156

Florenzano, M., \& Le Van, C. (2001). Finite Dimensional Convexity and Optimization. Springer-Verlag, Berlin, Heidelberg. https://doi.org/10.1007/978-3-642-56522-9

Giorgi, G. (1985). Programmi tangenti di programmi non lineari, Atti IX Convegno A.M.A.S.E.S., Levico Terme 3-5 Ottobre 1985, Pitagora Editrice, Bologna, 171-185.

Giorgi, G., \& Guerraggio, A. (1992). On the notion of tangent cone in mathematical programming, Optimization, 25, 11-23. https://doi.org/10.1080/02331939208843804

Giorgi, G., \& Guerraggio, A. (1994). First order generalized optimality conditions for a programming problem with a set constraint; in S. Komlosi, T. Rapcsàk and S. Schaible (Eds.), Generalized Convexity, Springer Verlag, Berlin, 171-185. https://doi.org/10.1007/978-3-642-46802-5_15

Giorgi, G., \& Guerraggio, A. (2002). Characterizations, comparisons, algebraic and topological properties of tangent cones, Journal of Statistics $\mathcal{E}$ Management Systems, 5, 275-294. https://doi.org/10.1080/09720510.2002.10701060

Gould, F. J., \& Tolle, J. W. (1971). A necessary and sufficient qualification for constrained optimization. SIAM J. Appl. Math., 20, 164-172. https://doi.org/10.1137/0120021

Guignard, M. (1969). Generalized Kuhn-Tucker conditions for mathematical programming problems in a Banach space. SIAM J. on Control, 7, 232-241. https://doi.org/10.1137/0307016

Hestenes, M. R. (1966). Calculus of Variations and Optimal Control Theory. J. Wiley, New York.

Hestenes, M. R. (1975). Optimization Theory. The Finite Dimensional Case. J. Wiley, New York.

Hiriart-Urruty, J.-B. (1979). Tangent cones, generalized gradients and mathematical programming in Banach spaces, Mathematics of Operations Research, 4, 79-97. https://doi.org/10.1287/moor.4.1.79

Hiriart-Urruty, J.-B., \& Lemarechal, C. (1993). Convex Analysis and Minimization Algorithms. Vol. I and Vol. II, Springer-Verlag, Berlin, Heidelberg, New York. https://doi.org/10.1007/978-3-662-02796-7

Jeter, M. W. (1986). Mathematical Programming. An Introduction, Marcel Dekker, Inc., New York.

Mangasarian, O. L. (1969). Nonlinear Programming. McGraw-Hill, New York.

Martein, L. (1985). Regularity conditions for constrained extremum problems, J. Optim. Theory Appl., 47, 219-233. https://doi.org/10.1007/BF00940770

Palata, J. (1989). A survey of conical approximations used in optimization. Optimization, 20, 147-161. https://doi.org/10.1080/02331938908843424

Polyak, B. T. (1987). Introduction to Optimization (Translations Series in Mathematics and Engineering). Optimization Software, Inc., Publications Division, New York.

Rockafellar, R. T. (1970). Convex Analysis, Princeton Univ. Press, Princeton. https://doi.org/10.1515/9781400873173

Rockafellar, R. T., \& Wets, R. J.-B. (2009). Variational Analysis (Corrected 3rd printing). Springer Verlag, Berlin.

Still, G., \& Streng, M. (1996). Optimality conditions in smooth nonlinear programming. J. Optim. Theory Appl., 90, 
483-515. https://doi.org/10.1007/BF02189792

Varaiya, P. P. (1967). Nonlinear programming in Banach spaces, SIAM J. Appl. Math., 15, 284-293. https://doi.org/10.1137/0115028

\section{Copyrights}

Copyright for this article is retained by the author(s), with first publication rights granted to the journal.

This is an open-access article distributed under the terms and conditions of the Creative Commons Attribution license (http://creativecommons.org/licenses/by/4.0/). 